Full-Length Article

\title{
Perceptions of Patients and Families who Received a Music Intervention During Mechanical
}

\section{Ventilation}

\author{
Mary F. Tracy ${ }^{1}$, Abbey Staugaitis ${ }^{2}$, Linda Chlan ${ }^{3}$, Annie Heiderscheit ${ }^{4}$ \\ ${ }^{1}$ University of Minnesota Medical Center, Minneapolis, Minnesota, USA \\ ${ }^{2}$ University of Minnesota, School of Medicine, Minneapolis, Minnesota, USA \\ ${ }^{3}$ College of Nursing, The Ohio State University, Columbus, Ohio, USA \\ ${ }^{4}$ Augsburg College, Minneapolis, Minnesota, USA
}

\begin{abstract}
The intensive care unit (ICU) is a technologically-driven environment where critically ill patients and their families have significant physical and emotional experiences. Mechanically ventilated (MV) patients can experience significant distress from anxiety and pain. Music listening is one integrative intervention that has been shown to reduce anxiety as well as other symptoms that contribute to distress in MV patients. This is a report of MV patient and family experiences from a larger research study aiming to evaluate levels of anxiety and sedative exposure with use of a patient-directed music intervention. Understanding perceptions of MV patients and families regarding the effectiveness of music listening might serve as a useful guide to improvement of their care.
\end{abstract}

Keywords: music intervention, mechanical ventilation, anxiety, pain, critical multilingual abstract $\mid$ mmd.iammonline.com care

\section{Introduction}

The intensive care unit (ICU) is a technologically-driven environment where the physical environment is one of little day/night differentiation [1]; elevated noise levels; painful procedures; and lack of privacy. Mechanically ventilated (MV) ICU patients can have long lengths of stay and experience significant distress - due to their physical condition and environment as well as from many therapeutic interventions that preclude ease of communication and a sense of control. Patients can suffer considerable anxiety and discomfort [1] and may develop a "form of post-traumatic stress disorder [2,3]." In an effort to alleviate symptoms of anxiety and pain and promote synchrony with mechanical breaths, nurses administer pain and sedative medications $[4,5]$; however, this can result in oversedation, loss of control, and difficulty weaning patients from MV [1]. ICU nurses may

\section{PRODUCTION NOTES: Address correspondence to:}

Mary Fran Tracy, PhD, RN, APRN, CNS, FAAN, University of Minnesota Medical Center, PCU 4C, 500 Harvard St. SE, Minneapolis, MN 55455, E-mail: Mtracy1@fairview.org | COI statement: Supported in part by a grant from the National Institute of Nursing Research, National Institutes of Health 1R01 NR 009295 (L. Chlan, Principal Investigator). The authors have no conflict of interest to declare. believe that sedated patients will not experience distress nor remember their critical care experience, however, that perception has been disputed [6]. Therefore simply sedating patients 'through' their ICU stay may actually worsen emotional sequelae and prolong physiological recovery from critical illness.

Researchers are exploring interventions that might promote comfort while maintaining the ability to keep MV patients interactive in their care. Music listening is one integrative intervention that has been shown to reduce anxiety as well as other symptoms that contribute to distress in MV patients [7]. While integrative therapies are increasingly reported to be of potential use in these situations, there is little data on critical care patient or family views of these therapies. This is a report of MV patient and family experiences from a larger research study [7] whose aim was to evaluate levels of anxiety and sedative exposure with use of a patient-directed music (PDM) intervention. Understanding perceptions of $\mathrm{MV}$ patients and families regarding the effectiveness of music listening will guide improvement of their care.

\section{Background}

The number of patients who recall the ICU experience ranges from 15 - 33\% [8-10]. MV patients have reported a wide array of distressing symptoms including pain, anxiety, not "getting enough air," fear of weaning, sleep disturbances, inability to communicate, loneliness, and helplessness [1,8,11-13]. Of 
these, one of the most distressing patient-reported symptoms is moderately intense anxiety [13].

Anxiety is a highly variable perception and may or may not decrease over time of ventilator support [11]. Patients may experience anxiety and depression 12 months after their ICU stay [8]. Some of this anxiety may be related to lighter sedation levels currently used in ICUs [1]; patients may be more cognizant of unclear thinking, resulting in an increased sense of loss of control [1]. They may also resist being sedated in trying to maintain clarity of thinking [14].

Family members have expressed a sense of "feeling connected" with their loved one, experiencing suffering with the ICU patient. This can be particularly true when patients are struggling to breathe. Families have expressed a desire to protect and "shield the patient from the deep emotions of the experience [15]."

Johnson articulated the important role of family members; families want to be involved in the patient's care [12]. This desire supports the importance of engaging family members in use of integrative therapies to reduce patient anxiety. It is important to know what families also think of these therapies as they can promote or discourage use by the patient. Therefore determining the perceptions of MV patients and families regarding the use of music listening can provide crucial information on the feasibility of this intervention for care providers to optimize the critical care experience.

\section{Parent Study Background}

The primary aim of the parent randomized, controlled clinical trial was to determine the effects of preferred, relaxing music on anxiety self-management and sedative exposure in critically ill MV patients [7]. The study was approved by the Human Subject Committees of the academic institution and study sites.

MV patients were enrolled from 12 ICUs in 5 medical centers in the urban Midwest United States. Patients were approached for participation if they were consistently following commands, participating in their daily care, and could engage in the informed consent process. Patients were randomly assigned to one of three groups: 1) experimental group of PDM whereby subjects listened to preferred music, tailored by a board-certified music therapist whenever desired; 2) active control condition of noise-canceling headphones whereby patients wore headphones to block out ICU noise whenever desired; and 3) control group of usual ICU care. $\mathrm{CD} / \mathrm{MP} 3$ players with Maxell noise-cancellation headphones (NC II) were utilized for the study. Participants in Group 1 had music preferences assessed at study entry and daily by the music therapist who compiled CDs with headphones placed at the bedside for each patient. (see Appendix $A$ for detailed information on the music selection and instruction process.) Participants randomized to the experimental patient-directed music (PDM) listening group self-initiated listening to preferred, individualized music whenever and for as long as desired each day enrolled on protocol, with encouragement to listen at least twice daily for 30 minutes. Participants listened to preferred music whenever feeling anxious or desiring relaxation based on individual needs. Thus, participants listened to music for varying times each study day with a daily mean of $79.8( \pm 126)$ minutes [7]. Refer to the manuscript describing the data capture with the data loggers for additional information [16].

Anxiety was assessed daily with a $100-\mathrm{mm}$ visual analog scale-anxiety. Patients remained in the study as long as they were receiving MV support, up to a 30-day protocol maximum. A total of $373 \mathrm{MV}$ patients were enrolled on protocol [7].

\section{Methods}

Research nurses visited participants daily during their enrollment in the parent study. Spontaneous comments from MV patients and family members about the use of the interventions or their study experience were recorded after each visit. On completion of the parent study, these field notes were collated. 2 members of the research team reviewed and independently categorized the documented patient and family comments into themes, finalizing when necessary by consensus.

\section{Results}

\section{Demographics}

Participants $(n=59)$ who provided comments regarding their study experiences comprised a sub-sample of $54 \%$ female with a mean age of 57 years ( \pm 14.6$) ; 78 \%$ Caucasian, 20\% AfricanAmerican, and 2\% Native American. Participants were in the ICU for a mean total of 21 days $( \pm 15.6)$ and received MV support for a mean total of 12.3 days $( \pm 11.4)$. The primary indication for MV in a majority of this sample was respiratory failure (48\%) or respiratory distress (33\%).

\section{Patient Experience Themes}

The most common remarks for both the music and headphone groups were reports of improved sleep resulting from blocking out the noise, which was particularly welcomed at night. One patient reported that the use of headphones was the only reason he was able to sleep and asked if he could take the headphones home. Many participants commented that the ability to block out ICU noise also helped them relax when they were awake.

PDM participants often expressed enjoyment and comfort from listening to preferred music. One patient reported improved attitude while listening to music and 
another said the music helped him have a more positive frame of mind. Patients reported that the music was helpful to "get through" procedures or pass time (see Table 1).

\section{Improved Sleep}

- Patient commented he is using the music at night and his sleep has improved. He feels he is sleeping better.

- Patient said he thinks the music is really helping him to relax and sleep at night.

- Patient enjoyed music and felt it helped her rest and relax.

- Patient was sleeping with headphones on. (Observed by research assistant)

- It (music) helped him relax, sleep, and even pass the time

- Patient stated that headphones (to block ICU noise) was "the best idea ever"

- Patient communicated that he liked to wear the headphones to help him get to sleep at night.

- Patient communicated that they (headphones) helped her rest despite the commotion in the next bed.

- Patient communicated that she wore the headphones to help her rest and that the headphones were comfortable to wear.

- Patient indicated she really likes the headphones. She wore them to help her sleep during dialysis.

Enjoyment and Comfort

- Patient listening to Johnny Cash and mouthed the word "singing"

- Patient shared that he is enjoying the music.

- Patient commented on how the music lowered his blood pressure and how relaxed he was when he awoke.

- Patient said it (music) is wonderful and that having the music made a big difference and helped his attitude

- Patient said he really liked the music and listening with headphones was "relaxing" and "helped" him.

- Patient tapping fingers to the music provided to him.

- Nurse said patient always nods "yes" when asked if he would like to wear the headphones.

- Patient talked a lot about wanting to dance across the room in his chair.

- "I really enjoy the music. It is helpful."

- "Music makes me feel better."

- "The music gal went above and beyond finding the perfect music."

- "The music was relaxing and helped."

Gratitude

- Patient thanked the research assistant "a few times" for being part of the study. Subject also wished to thank music therapist for bringing him the music he liked.

- Patient was appreciative of the study

- Patient thanked us for the music.

- Patient thanked the research assistant "and you girls" for doing the study.

- Patient signaled "thumbs up" for the music.

- Patient gave "thumbs up" for the study experience.

Table 1. Responses from Mechanically Ventilated Patients Receiving Music Intervention
A third general theme that emerged was gratitude. PDM and headphone participants often stated that they were thankful for the interventions and expressed gratitude for the opportunity to participate in the study. Two patients gave "thumbs up" when asked what they thought of their experience with the study.

\section{Family Experience Themes}

Family members also reported that blocking noise improved and increased sleep and relaxation for their MV patient. One spouse purchased headphones for his wife to help her continue to block out noise after she completed the study.

Family members of PDM participants stated they believed the patient enjoyed listening to music. One spouse commented that the patient listened to music all the time. Another family commented that they felt the music made the patient feel better.

Family members also expressed gratitude for the interventions and the opportunity to participate in research. One participant's daughter said that she was proud of her father for participating (see Table 2).

\section{Improved Sleep}

- Patient's husband said, "The headphones helped her so much that I went out and bought her some Bose headphones so that she could use them when she wanted to get some rest."

\section{Enjoyment and Comfort}

- "She had a great selection of music that she liked and seemed to really enjoy listening to it."

- "She requested to listen to the music often."

- "He listens all the time."

- Patient's wife says he listens to the music all of the time and it has been working well.

Gratitude

- "I was so impressed with the process. Thank you so much."

- "It's a great study."

- "The music helped her so much. She got better so quickly after she was able to listen to the music. Maybe music and prayers are the trick."

- Daughter of family said she was proud of her father for participating in this study.

- Wife of patient verbalized how "wonderful" this type of research is and thinks her husband's participation is very important.

Table 2. Responses from Family Members of Mechanically Ventilated Patients Receiving Music Intervention 


\section{Discussion}

MV patients who used PDM reported benefits of relaxation, improved sleep and improved outlook. This was consistent with observational reports from family members who commented that patients appeared to be less anxious and slept better with music intervention. Patients perceived benefits from music listening and were grateful for the opportunity to use this integrative therapy.

There is increasing evidence that a significant number of patients develop PTSD after ICU stays [17-19]. Risk factors identified for development have been shown to be agitation and extreme fear [19], frightening memories of ICU experiences [17], and high use of benzodiazepine sedatives $[17,18]$. This has led to research to minimize or eliminate altogether use of sedative medications in MV patients [20]. Appropriate use of sedatives continues to be important, however, we cannot ignore MV patients' anxiety. There is great need to provide alternatives/adjuncts to sedatives in anxiety management. There is growing evidence that use of integrative interventions such as music can play an essential role in decreasing use of sedatives and improving levels of anxiety in MV patients [7]. While the themes provided here are only initial steps in exploring patient and family perspective on use of PDM, they provide a foundation for continued research on patient-centered outcomes for this therapy.

\section{Limitations}

Comments gathered from patients and family members were documented as they were offered during parent study participation. This data was not specifically gathered as a formal qualitative study with the sole purpose of determining patient and family perspectives. Further research is needed to delineate all MV patient and family perspectives about use of $\mathrm{PDM}$ and its potential to improve the ICU experience.

\section{Conclusions}

Comments indicate MV patients and family members perceive benefits from listening to music and are grateful for the opportunity to use this integrative therapy. As increasing emphasis is placed on improving the patient experience, it behooves care providers to utilize all feasible interventions such as music listening to optimize care.

\section{References}

1. Schou L, Egerod I. A qualitative study into the lived experience of postCABG patients during mechanical ventilator weaning. Intens Crit Care Nurs. 2008: 24:171-179.

2. Bench S, Day T. The user experience of critical care discharge: a metasynthesis of qualitative research. Int J Nurs Stud. 2009: 47:487-499.
3. Griffiths RD, Jones C. Delirium, cognitive dysfunction, and posttraumatic stress disorder. Curr Opin Anaesth. 2007: 20:124-129.

4. Guttormson JL, Chlan L, Weinert C, Savik K. Factors influencing nurse sedation practices with mechanically ventilated patients: a U.S. national survey. Intens Crit Care Nurs. 2010: 26(1):44-50.

5. Weinert CR, Calvin AD. Epidemiology of sedation and sedation adequacy for mechanically ventilated patients in a medical and surgical intensive care unit. Crit Care Med. 2007: 35:393-401.

6. Lof L, Berggren L, Ahlstrom G. Severely ill ICU patients recall of factual events and unreal experiences of hospital admission and ICU stay- 3 and 12 months after discharge. Intens Crit Care Nurs. 2006: 22:154-166.

7. Chlan LL, Weinert CR, Heiderscheit A, et al. Effects of patient directed music intervention on anxiety and sedative exposure in critically ill patients receiving mechanical ventilation support: a randomized clinical trial. JAMA. 2013: 309(22):2330-44.

8. Rattray J, Crocker C, Jones M, Connaghan J. Patients' perceptions of and emotional outcome after intensive care: results from a multicentre study. Nurs Crit Car. 2010: 15:86-93.

9. Ringdal M, Johansson L, Lundberg D, Bergbom I. Delusional memories from the intensive care unit - Experienced by patients with physical trauma. Intens Crit Care Nurs. 2006: 22(6):346-64.

10. Samuelson K, Lundberg D, Fridlund B. Memory in relation to depth of sedation in adult mechanically ventilated intensive care unit patients. Intens Care Med. 2006: 2:660-67.

11. Chlan L, Savik K. Patterns of anxiety in critically ill patients receiving mechanical ventilatory support. Nurs Res. 2011: 60:S50-S57.

12. Johnson P. Reclaiming the everyday world: how long-term ventilated patients in critical care seek to gain aspects of power and control over their environment. Intens Crit Care Nurs. 2004: 20:190-199.

13. Li D, Puntillo K. A pilot study on coexisting symptoms in intensive care patients. App Nurs Res. 2006: 19:216-19.

14. Egerod I. Uncertain terms of sedation in ICU. How nurses and physicians manage and describe sedation for mechanically ventilated patients. J Clin Nurs. 2002: 11: 831-840.

15. Eggenberger S, Nelms TP. Being family: the family experience when an adult member is hospitalized with a critical illness. J Clin Nurs. 2007: 16:1618-1628

16. Chlan L, Patterson B, Heiderscheit A. Data acquisition for a patientdirected protocol in the dynamic intensive care unit setting. Contemporary Clinical Trials. 2011: 32: 544-546.

17. Davydow DS, Gifford JM, Desay SV, Needham DM, Bienvenu JO. Posttraumatic stress disorder in general intensive care unit survivors: a systematic review. Gen Hosp Psych. 2008: 30:21-434.

18. Jones C, Backman C, Capuzzo M, Flaatten H, Rylander C, Griffiths RD. Precipitants of post-traumatic stress disorder following intensive care: a hypothesis generating study of diversity in care. Intens Care Med. 2007: 33:978-985.

19. Samuelson K, Lundberg D, Fridlund B. Stressful memories and psychiatric distress in adult mechanically ventilated intensive care patients - a 2-month follow up study. Acta Anaesth Scand. 2007: 51:671-678.

20. Kress JP, Hall JB. Sedation in the mechanically ventilated patient. Crit Care Med. 2006: 34:2541-2546.

21. Chlan L, Heiderscheit A. A tool for music preference assessment in critically ill patients receiving mechanical ventilatory support: an interdisciplinary approach. Music Therapy Perspectives. 2009: 27(1):4247.

22. Heiderscheit A, Breckenridge S, Chlan L, Savik K. Music preferences of mechanically ventilated patients participating in a randomized controlled trial. Music and Medicine. 2014: 6(2):29-38. 


\section{Appendix A}

\section{Patient Directed Music Listening Protocol}

A board certified music therapist assessed patient music preferences utilizing the Music Assessment Tool (MAT) developed by Chlan and Heiderscheit [21,22]. Utilizing the MAT allowed patients to indicate their preferred music genres, groups, artists, and instrumentation. Additionally, patients were also able to indicate specific genres, groups or artists they did not prefer. This permitted patients to identify nuances within their preferences. For example, if they enjoyed listening to country music, but did not like a particular country artist or group, this could be identified and delineated in the assessment process. The music therapist utilized the patient preferences as well the study determinant of 60-80 beats per minute to identify the music for each patient.

Following the music preferences assessment, the music therapist burned all the music the patient identified as their preferred music onto CDs. Patients were able listen to the music whenever they wanted to and as long as they wanted to throughout their enrollment in the study. The music therapist met with patients on a daily basis to continue to assess and identify any additional music they wanted during the course of the study. Throughout their enrollment in the study, patients could identify and request music.

Patients enrolled in the study selected music from 14 genres included on the MAT. These genres included blues, reggae, classical, country, jazz, new age, oldies (1940-1970), pop music, hip hop, rap, rhythm and blues, religious/sacred, rock and world music [22]. Patients listened to the music via a
MP3 player with noise cancelling headphones [7]. Patients listening time was recorded via a data logger that was attached to the noise headphones and MP3 player [16].

Heiderscheit et al. [22] reported that during the study $1,580 \mathrm{CDs}$ were distributed to the 126 patients in the music group. This resulted in patients receiving a median of $13 \mathrm{CDs}$ (range 0-56). The researchers also reported that patients listened to music a median of 63.8 minutes per day [22].

\section{Biographical Statements}

Mary Fran Tracy, PhD, RN, APRN, CNS, FAAN. Dr. Tracy is a Critical Care Clinical Nurse Specialist at the University of Minnesota Medical Center.

Abbey Staugaitis, MS, RN. Ms. Staugaitis is a Clinical Research Coordinator with the Neurological Emergency Treatment Trials Network (NETT) located at the University of Minnesota.

Linda Chlan, PhD, RN, FAAN. Dr. Chlan is Distinguished Professor of Symptom Management Research Center of Excellence in Critical \& Complex Care and Associate Director \& Director of Symptom Science at the The Ohio State University.

Annie Heiderscheit, PhD, MT-BC, LMFT. Dr. Heiderscheit is an Assistant Professor and Director of the Master of Music Therapy Program at Augsburg College, Minneapolis, MN. 